\title{
Higher order approximation of the period-energy function for single degree of freedom Hamiltonian Systems
}

\author{
Silvia Foschi, Giovanni Mingari Scarpello and Daniele Ritelli *
}

\begin{abstract}
In 1985 Franz Rothe [16] found, by means of the thermodynamical equilibrium theory, an asymptotic estimate of period of solutions of Ordinary Differential Equations originated by predator - prey Volterra - Lotka model. We extend some of Rothe's ideas to more general systems:

$$
\left\{\begin{array}{l}
\dot{p}(t)=-g(q(t)) \\
\dot{q}(t)=f(p(t))
\end{array}\right.
$$

and succeed in calculating the period's asymptotic analytic expression as a function of the energy level. We finally check our result re-obtaining classical period's estimation of some popular Hamiltonian systems. We apply our technique also to a nonlinear Hamiltonian system whose period is not available in the literature.
\end{abstract}

KEYWORD: Hamiltonian systems, series reversion, period, asymptotic expansion

NOTE: this is the post print version of the paper appeaered on Meccanica 39 (2004) 357-368

doi: 10.1023/B:MECC.0000029367.00112.82

\section{Introduction}

This paper supplies an asymptotic expansion of the energy-period function for planar systems like:

$$
\left\{\begin{array}{l}
\dot{p}(t)=-\frac{\partial}{\partial q} H(p(t), q(t)), \\
\dot{q}(t)=\frac{\partial}{\partial p} H(p(t), q(t)) .
\end{array}\right.
$$

They are characterized by an uncoupled Hamiltonian $H \in \mathcal{C}^{\infty} H(p, q)=F(p)+G(q)$, being $F(p)$ and $G(q)$ some non negative and analytical functions of momentum $p$ and of the lagrangian sole coordinate $q .{ }^{1}$ Furthermore both $F(p)$ and $G(q)$ take the value zero at the origin where have a non-degenerate minimum. This may be ensured asking that:

$$
F^{\prime}(0)=G^{\prime}(0)=0 \text { and } F^{\prime \prime}(0), G^{\prime \prime}(0)>0 .
$$

We will suppose (2) satisfied throughout all our treatment. Nevertheless it is possible, via some adjustments, to keep valid the forthcoming conclusions even if the minimum's order of almost one term of the uncoupled hamiltonian is greater than two. I.e. if $n \in \mathbb{N}, n>1$ exists such that:

$$
F^{\prime}(0)=F^{\prime \prime}(0)=\cdots=F^{(2 n-1)}(0)=0, \text { and } F^{(2 n)}(0)>0 .
$$

Furthermore we will assume the functions $F(p)$ and $G(q)$ definite over intervals even unlimited, but symmetric to the origin, like $[-a, a]$ and $[-b, b]$ respectively. Under these assumptions the system (1) notoriously has a non-degenerate center at the origin which all the solutions are orbiting about. We are also admitting that Riemann integrals:

$$
z_{F}(\gamma)=\int_{-a}^{a} e^{-\gamma F(p)} d p, z_{G}(\gamma)=\int_{-b}^{b} e^{-\gamma G(q)} d q,
$$

\footnotetext{
${ }^{*}$ Dipartimento di Matematica per le scienze economiche e sociali, Università di Bologna daniele.ritelli@unibo.it

${ }^{1}$ This requirement is, for example, not met by the Van der Pol oscillator Hamiltonian due to a presence of a mixed term containing both $p$ and $q$.
} 
converge absolutely. Typically the $p$ variable means momentum, then $F(p)$ domain is the whole real axis. On the contrary, the $G(q)$ domain is depending upon the system's geometry and the field of forces which it is merged in. All these systems have an Hamiltonian like:

$$
H(p, q)=\frac{1}{2 m} p^{2}+G(q)
$$

Several features of system (1) have been tackled: e. g. in [4] the inverse problem is solved to find those Hamiltonian systems having a given period function $T(h)$. On the contrary, in [5] the isochronism is studied, i.e. $T(h)=$ constant.

The monotonicity of period function has been often considered: see [18], [16], [17] and, more recently, [7] and [2]. A great effort has been made in order to detect asymptotic approximations for period function, [16], [17], [9], [10] and [19]. To compute the period of solutions of (1) can be useful also to delayed differential equations analysis of Kaplan-Yorke type, [11] :

$$
\dot{x}(t)=-g(x(t-1)),
$$

where $g: \mathbb{R} \rightarrow \mathbb{R}$ is odd and:

$$
x g(x)>0 \text { for } x \neq 0 \text { and } g(0)=0 .
$$

Let us fix a bit of terminology, following [16] and [4]. We will start with the function $(t, h) \in \mathbb{R} \times[0,+\infty) \rightarrow$ $(p, q) \in \mathbb{R}^{2}$ where the couple $(p(t), q(t))$ is the (1) solution such that $q(0, h)=0, p(0, h) \geq 0$ and for each $(t, h) \in \mathbb{R} \times[0,+\infty):$

$$
H(p(t, h), q(t, h))=h \text {. }
$$

The notation $p(t, h)$ and $q(t, h)$ means that to each energy level $h$, one and only one solution there will be to (1). Furthermore the period function is utilized $T: h \in[0,+\infty) \rightarrow T(h) \in(0,+\infty)$ for each $(t, h) \in \mathbb{R} \times[0,+\infty)$ defined by:

$$
p(t+T(h), h)=p(t, h), \quad q(t+T(h), h)=q(t, h),
$$

where $T(h)$ is the fundamental period, namely the smallest positive number expressing a periodicity linked to the energy level $h$. Rothe introduced in [16] and [17] the aforementioned concepts in the case of Volterra - Lotka equations, where $F(p)=c\left(e^{p}-p-1\right)$ and $G(q)=a\left(e^{q}-q-1\right)$. He established an asymptotic expansion theorem ${ }^{2}$ concerning period function. We mean to generalize that theorem.

Rothe's Theorem is (see 1985, Lemma 3 page 133):

${ }^{2}$ The asymptotic expansions took their origin in such a way. If one tries to solve the ordinary differential equation:

$$
y^{\prime}+y=\frac{1}{x}
$$

for large $x$, and looks for a function of the form:

$$
y(x)=\sum_{m=1}^{\infty} a_{m} x^{-m}
$$

he will find (see [14], page 6):

$$
y(x)=\frac{0 !}{x^{1}}+\frac{1 !}{x^{2}}+\frac{2 !}{x^{3}}+\frac{3 !}{x^{4}}+\cdots
$$

This series, using e.g. the ratio test, is found to diverge for all values of $x$. Truncating it after $n \in \mathbb{N}$ terms, the remainder $R_{n}(x)$ will be a function of both $n$ and $x$ such that $R_{n}(x) \rightarrow \infty$ for $n \rightarrow \infty$ for any $x$.

But fixing $n$, the truncation error after $n$ terms is numerically less than first $(n+1)^{\text {th }}$ neglected term. Such a series, even if considered by Euler (1707-1783), Laplace (1749-1827), Puiseux (1820-1883), Borel (1871-1956) and others, is referred as asymptotic series of Poincaré type (H. Poincaré (1854-1912): Les méthodes nouvelles de la mécanique céleste, 1893, II, ch. VII.) E. Borel, in a paper published in the VI volume (1899) of the Annales de l'Ecole Normale Superieure, defined the absolute summability of a divergent series and established by theorem how this property enables to handle the series, exactly as if it were a convergent one.

The relationship between the asymptotic expansion and $y(x)$ is written as:

$$
y(x) \sim \frac{0 !}{x^{1}}+\frac{1 !}{x^{2}}+\frac{2 !}{x^{3}}+\frac{3 !}{x^{4}}+\cdots
$$

The right hand side series is asymptotic to $y(x)$ in the neighbourhood of $x \rightarrow \infty$.

It is useful to underline that the above definition has been generalized: in such a way a series of the type:

is said to be asymptotic to $y(x)$ in $x=0$, if:

$$
a_{0}+a_{1} x+a_{2} x^{2}+a_{3} x^{3}+\ldots+a_{j} x^{j}+\cdots
$$

$$
\lim _{x \rightarrow 0} \frac{1}{x^{n}}\left[y(x)-\sum_{j=0}^{n-1} a_{j} x^{j}\right]=0 .
$$


Theorem 1.1. The energy-period $T(h)$ has the asymptotic expansion:

$$
T(h) \sim a_{0}+a_{1} h+a_{2} \frac{h^{2}}{2}+a_{3} \frac{h^{3}}{3 !}+\cdots
$$

where:

$$
\begin{aligned}
& a_{0}=\frac{2 \pi}{\sqrt{a c}} \\
& a_{1}=\frac{\pi}{6 \sqrt{a c}}\left(\frac{1}{a}+\frac{1}{c}\right), \\
& a_{2}=\frac{\pi}{144 \sqrt{a c}}\left(\frac{1}{a}+\frac{1}{c}\right)^{2}, \\
& a_{3}=-\frac{139 \pi}{25,920 \sqrt{a c}}\left(\frac{1}{a}+\frac{1}{c}\right)\left(\frac{1}{a^{2}}+\frac{1}{c^{2}}-\frac{154}{139} \frac{1}{a c}\right) .
\end{aligned}
$$

The following observations are valid for both: Theorem (1.1) and our successive work:

- in general one cannot establish convergency for the Maclaurin series of the energy - period function asymptotically expanded in the sense of Poincaré;

- asymptotic expansion can be obtained only for small oscillations performed at low energy levels. As we shall see later, this restriction about validity range of the approximation, is depending on Laplace reverse transform of asymptotic expansions of integrals like (3). Such asymptotic espansions are valid for large values of the argument object of the reverse transform;

- Rothe made an asymptotic expansion of Volterra-Lotka system driven by that of the logarithm of Gamma function, ${ }^{3}$ see [20] pp. 251-253. In our more general approach we will construct our asymptotic expansions following Laplace's method for the integrals, see [15] chapter 3 .

Our article's main result is:

Theorem 1.2. The energy-period function of a system (1) can be asymptotically espanded in this way:

$$
T(h) \sim \sum_{n=1}^{\infty} \zeta_{n} \frac{h^{n-1}}{(n-1) !}
$$

with:

$$
\zeta_{n}=\sum_{k=1}^{n} \alpha_{k} \beta_{k}
$$

where:

$$
\begin{aligned}
& \alpha_{k}=(2 k-1) \cdot f_{2 k-1} \cdot \Gamma\left(\frac{2 k-1}{2}\right), \\
& \beta_{k}=(2 n+1-2 k) \cdot g_{2 n+1-2 k} \cdot \Gamma\left(\frac{2 n+1-2 k}{2}\right),
\end{aligned}
$$

and the coefficients $f_{n}, g_{n}$ are obtained inverting the power series of $F(p)$ and $G(q)$, i.e.:

$$
\begin{aligned}
& F(p)=x \Rightarrow p \sim \sum_{n=1}^{\infty} f_{n} x^{\frac{n}{2}}, \\
& G(q)=x \Rightarrow q \sim \sum_{n=1}^{\infty} g_{n} x^{\frac{n}{2}} .
\end{aligned}
$$

We wish to stress that the symbol $\sum_{n=1}^{\infty}$ should be intended in sense of the asymptotic expansions. At introduction's closure, we highlight that the particular asymptotic expansion of the period function obtained in [16] was used for proving its monotonicity.

\footnotetext{
${ }^{3}$ The astronomers persevered in using divergent series, even if banned by Cauchy (1789-1857), urged by the necessities of their science. But by Cauchy himself these series were later deemed really helpful, and in a famous article (Sur l'emploi légitime des séries divergentes, 1843) he treated the Sterling series for calculating $\ln \Gamma(x), x>>1$. He also proved that, having fixed the number $n$ of terms, the absolute error in truncating the series after $n$ terms is less than the first neglected term, and decreases for $x$ rising.
} 


\section{The main result}

This section will show how Rothe's early approach has been extended to the general case:

$$
\left\{\begin{array}{l}
\dot{p}(t)=-g(q(t)) \\
\dot{q}(t)=f(p(t))
\end{array}\right.
$$

where:

$$
f(p)=\frac{d}{d p} F(p), g(q)=\frac{d}{d q} G(q) .
$$

Let us define the state sum function (or: canonical partition function) generated by the Hamiltonian system (6) for $\beta \in(0,+\infty)$ :

$$
Z(\beta)=\iint_{\mathcal{R}} e^{-\beta(F(p)+G(q))} d p d q .
$$

where $\mathcal{R}$ is the rectangle obtained as cartesian product of domains of $F(p)$ and $G(q)$. Such a definition coincides with that given in [16] for Volterra-Lotka system. Remembering (3) we can express (7) as:

$$
Z(\beta)=z_{F}(\beta) z_{G}(\beta),
$$

as a consequence of iterated integration. The relationship linking the state sum function to energy-period function is highlighted (see also [16] pag. 131 eq. (12) and [17] pag. 132 eq. (19)) by the:

Theorem 2.1. The state sum function is the Laplace transform of energy-period function $T(h)$.

Then the remarkable formula:

$$
Z(\beta)=\int_{0}^{+\infty} e^{-\beta h} T(h) d h,
$$

holds. The main problem to be solved in order to achieve a series development of energy-period function $T(h)$ is to construct an asymptotic espansion of integrals in (3). This can be accomplished following Laplace, as presented by [15]. ${ }^{4}$ He takes into account parametric integrals like:

$$
I(\gamma)=\int_{a}^{b} e^{-\gamma R(z)} d z,
$$

where $a, b, R(z)$ do not depend on the real parameter $\gamma>0, R \in \mathcal{C}^{\infty}$. If $R(a)=0$ and $R$ is analytic, with absolute minimum in $x=a$ and then $R^{\prime \prime}(a)>0$, Olver establishes:

Lemma 2.1. If $\gamma \rightarrow+\infty$, then the asymptotic expansion holds:

$$
\int_{a}^{b} e^{-\gamma R(z)} d z \sim \sum_{n=1}^{+\infty} \frac{n}{2} \Gamma\left(\frac{n}{2}\right) \frac{c_{n}}{\gamma^{\frac{n}{2}}}
$$

with $c_{n}$ computed by performing the reversion:

$$
R(z)=x \Longleftrightarrow z=\sum_{n=1}^{\infty} c_{n} x^{\frac{n}{2}}
$$

Proof. See [15], Theorem 8.1, pages 86-87.

\footnotetext{
${ }^{4}$ Laplace (Théorie analytique des probabilités, third edition, 1820), integrating iteratively by parts, obtained the error complementary function:

$$
\operatorname{Erfc}(T)=\int_{T}^{\infty} e^{-t^{2}} d t=\frac{e^{-T^{2}}}{2 T}\left[1-\frac{1}{2 T^{2}}+\frac{1 \cdot 3}{\left(2 T^{2}\right)^{2}}-\frac{1 \cdot 3 \cdot 5}{\left(2 T^{2}\right)^{3}}+\cdots\right],
$$

and, even if conscious of the divergent character of the series, he used it for evaluating $\operatorname{Erfc}(T)$, for $T>>1$.
} 
Once again each series should be intended as asymptotic expansion. We have to manage bilateral integrals: then it will be useful to decompose them. For instance the first integral in (3) can be written:

$$
\int_{0}^{a} e^{-\beta F(-p)} d p+\int_{0}^{a} e^{-\gamma F(p)} d p
$$

Then we have to revert not only $F(p)$, but also $F(-p)$. By inspecting reversion formula, we denote with $f_{n}^{+}$the reversion coefficients of $F(p)$ and with $f_{n}^{-}$the $F(-p)$ ones. Then, if $n \in \mathbb{N}$ is even, we have:

$$
f_{n}^{+}+f_{n}^{-}=0
$$

whilst, if $n \in \mathbb{N}$ is odd:

$$
f_{n}^{+}-f_{n}^{-}=0 \text {. }
$$

The reader is referred to [12] pages 187-188, where the change $p^{2}=x$ has been made. Of course the assumptions (2) imply that the asymptotic series so obtained is a Puiseux one. ${ }^{5}$ In this way we established the validity of:

Lemma 2.2. If $f_{n}$ and $g_{n}$ are the coefficients obtained reverting $F(p)$ and $G(q)$, then the integrals introduced in (3) allow the asymptotic expansions:

$$
\begin{aligned}
& z_{F}(\gamma) \sim \sum_{n=1}^{\infty}(2 n-1) f_{2 n-1} \Gamma\left(\frac{2 n-1}{2}\right) \gamma^{-\frac{2 n-1}{2}} \\
& z_{G}(\gamma) \sim \sum_{n=1}^{\infty}(2 n-1) g_{2 n-1} \Gamma\left(\frac{2 n-1}{2}\right) \gamma^{-\frac{2 n-1}{2}}
\end{aligned}
$$

Finally, let us go to the last Lemma necessary for Theorem (1.2).

Lemma 2.3. The state sum function (7) can be expanded asymptotically:

$$
Z(\beta) \sim \sum_{n=1}^{\infty} \zeta_{n} \beta^{-\frac{1}{n}}
$$

with:

$$
\zeta_{n}=\sum_{k=1}^{n}(2 k-1) f_{2 k-1} \Gamma\left(\frac{2 k-1}{2}\right)(2 n+1-2 k) g_{2 n+1-2 k} \Gamma\left(\frac{2 n+1-2 k}{2}\right) .
$$

Proof. Recalling Lemma (2.2) and observing that the asymptotic expansions can be seen as power series of odd degree in the variable $x=\gamma^{-\frac{1}{2}}$, we can use Cauchy's product formula for power series, obtaining:

$$
\sum_{n=1}^{\infty} \xi_{2 n-1} x^{2 n-1} \times \sum_{n=1}^{\infty} \eta_{2 n-1} x^{2 n-1}=\sum_{n=1}^{\infty} \zeta_{2 n} x^{2 n}
$$

where:

$$
\zeta_{2 n}=\sum_{k=1}^{n} \xi_{2 k-1} \eta_{2 n-(2 k-1)}
$$

Thesis then follows from (8).

Proof of Theorem (1.2). Proof now follows solely from a reverse Laplace transformation by Theorem (2.1).

\footnotetext{
${ }^{5}$ It was a Newton (1642-1727) remarkable discovery (De methodis serierum et fluxionum, 1671) that any algebraic function $y$ can be expressed as a fractional power series in $x$ :$$
y=a_{0}+a_{1} x^{r_{1}}+a_{2} x^{r_{2}}+\cdots
$$

where $r_{k}$ are rational numbers. For example if $y^{2}(1+x)^{2}=x$, we have:

$$
y=\frac{x^{\frac{1}{2}}}{1+x}=x^{\frac{1}{2}}\left(1-x+x^{2}-x^{3}+\cdots\right),
$$

in particular, near to the origin, there are two values of $y$ for each $x$. In such a way, a series expansion - different from the usual Maclaurin one - is capable of describing the function branching behaviour which, for small $x$, tends to square root of $x$, and then is a two-valued function. Newton contributed an ingenious algorithm for obtaining the successive powers of $x$. A more rigorous proof was given two centuries later by V. Puiseux in the memoir Recherches sur les fonctions algébriques (1850). For this reason the fractional power series expansion of algebraic functions is now called Puiseux expansion.
} 


\section{Applications}

Now we are going to expand asymptotically the period function of well known Hamiltonian systems. For the elementary case of the linear oscillator we have:

$$
H(p, q)=\frac{1}{2 m} p^{2}+\frac{1}{2} a q^{2} ; a, m>0 .
$$

Its period function is a constant, which we will find as a special case of Theorem (1.2). is:

Successively we will obtain the results of [16] concerning the Volterra-Lotka equations, whose Hamiltonian

$$
H(p, q)=a\left(e^{p}-p-1\right)+c\left(e^{q}-q-1\right) ; a, c>0 .
$$

We cannot neglect mathematical models whose period function depends on complete elliptic integrals, like the mathematical pendulum, whose Hamiltonian is:

$$
H(p, q)=\frac{1}{2 m} p^{2}+\frac{g}{\ell}(1-\cos q) ; m, g, \ell>0,
$$

or the Duffing nonlinear oscillator:

$$
H(p, q)=\frac{1}{2 m} p^{2}+\frac{1}{2} q^{2}+\frac{1}{4} \varepsilon q^{4} ; m, \varepsilon>0 .
$$

In last two systems we will compare our asymptotic expansion with those obtained expanding the complete elliptic integrals giving the periods.

Finally, we will apply our Theorem to further more complicated Hamiltonian systems whose period cannot be expressed even by special functions. Such a Hamiltonian function:

$$
H(p, q)=\frac{1}{2 m} p^{2}+\left(\frac{1-2 b}{2}-\frac{-2 b-2 b q^{2}+\sqrt{1+q^{2}}+q^{2} \sqrt{1+q^{2}}}{2 \sqrt{1+q^{2}}}\right) \omega^{2},
$$

$m, b>0$ models the behaviour of a single degree of freedom system - presented in [1] pag. 10 - made of a particle moving along a fixed straightline and under the elastic force of a wire fixed to another point not belonging to trajectory. The system's troubleness depends on the geometry only, being there neither weight, nor reactions, nor drag.

\subsection{The linear oscillator}

This very simple system is treated here for achieving an easy test of the Theorem (1.2). Here the reversion procedure is straightforward:

$$
F(p)=\frac{1}{2 m} p^{2}=x \Rightarrow p=(2 m)^{\frac{1}{2}} x^{\frac{1}{2}}
$$

then the only nonzero term coming from the reversion is: $f_{1}=(2 m)^{\frac{1}{2}}$. Analogously:

$$
G(q)=\frac{1}{2} a q^{2}=x \Rightarrow q=\left(\frac{2}{a}\right)^{\frac{1}{2}} x^{\frac{1}{2}},
$$

and then $g_{1}=\left(\frac{2}{a}\right)^{\frac{1}{2}}$. Therefore applying formulas (4) and (5) we have:

$$
T(h)=\left(\Gamma\left(\frac{1}{2}\right)\right)^{2} f_{1} \cdot g_{1}=\pi(2 m)^{\frac{1}{2}}\left(\frac{2}{a}\right)^{\frac{1}{2}}=2 \pi\left(\frac{m}{a}\right)^{\frac{1}{2}} .
$$

The above formula allows to compute, from a very general point of view, the period of the second order linear differential equation:

$$
\ddot{p}(t)+\frac{a}{m} p(t)=0 .
$$

Of course the physical meaning of equation implies $m>0$ and $a>0$. 


\subsection{The Volterra - Lotka system}

The Volterra - Lotka predator-prey system is quite different from the other ones considered hitherto, due to its not properly mechanical nature. Let us carry out reversion of $F(p)=a\left(e^{p}-p-1\right)$ and of $G(q)=$ $c\left(e^{q}-q-1\right)$ for obtaining $f_{2 n-1}$ and $g_{2 n-1}$ of Lemma 2.2. Otherwise speaking, we have to evaluate Puiseux series generated reverting both functions $\widetilde{F}(p)=F(p)+F(-p)$ and $\widetilde{G}(q)=G(q)+G(-q)$. Computer Algebra plays an essential role here, because the relevant symbolic computations would require several days of work without it. Reverting $\widetilde{F}(p)=x$ we obtain:

$$
\begin{aligned}
p= & 2 \sqrt{2}\left(\frac{x}{a}\right)^{\frac{1}{2}}+\frac{\sqrt{2}}{9}\left(\frac{x}{a}\right)^{\frac{3}{2}}+\frac{1}{270 \sqrt{2}}\left(\frac{x}{5}\right)^{\frac{5}{2}}+ \\
& -\frac{139}{170,100 \sqrt{2}}\left(\frac{x}{a}\right)^{\frac{7}{2}}-\frac{571}{36,741,600 \sqrt{2}}\left(\frac{x}{a}\right)^{\frac{9}{2}}+ \\
& +\frac{163,879}{16,974,619,200 \sqrt{2}}\left(\frac{x}{a}\right)^{\frac{11}{2}}+\cdots
\end{aligned}
$$

Analogously from $\widetilde{G}(q)=x$ we get:

$$
\begin{aligned}
q= & 2 \sqrt{2}\left(\frac{x}{c}\right)^{\frac{1}{2}}+\frac{\sqrt{2}}{9}\left(\frac{x}{c}\right)^{\frac{3}{2}}+\frac{1}{270 \sqrt{2}}\left(\frac{x}{5}\right)^{\frac{5}{2}}+ \\
& -\frac{139}{170,100 \sqrt{2}}\left(\frac{x}{c}\right)^{\frac{7}{2}}-\frac{571}{36,741,600 \sqrt{2}}\left(\frac{x}{c}\right)^{\frac{9}{2}}+ \\
& +\frac{163,879}{16,974,619,200 \sqrt{2}}\left(\frac{x}{c}\right)^{\frac{11}{2}}+\cdots
\end{aligned}
$$

Therefore, applying formulas (4) and (5), $T(h)$ asymptotic espansion is found to be:

$$
\begin{aligned}
T(h) \sim & 2 \pi(a c)^{-\frac{1}{2}}+\frac{a+c}{6} \pi(a c)^{-\frac{3}{2}} h+\frac{(a+c)^{2}}{288} \pi(a c)^{-\frac{5}{2}} h^{2}+ \\
& +\frac{a+c}{155,520}\left(-139 a^{2}+154 a c-139 c^{2}\right) \pi(a c)^{-\frac{7}{2}} h^{3}+ \\
& +\frac{(a+c)^{2}}{29,859,840}\left(-571 a^{2}+586 a c-571 c^{2}\right) \pi(a c)^{-\frac{9}{2}} h^{4}+\cdots
\end{aligned}
$$

With a little algebra the results of Rothe [16] are found - see theorem (1.1) - concerning terms of degree $\leq 3$ with respect to the energy level $h$.

\subsection{The mathematical pendulum}

The pendulum's free movement differential equation with initial rest is:

$$
\left\{\begin{array}{l}
\ddot{\vartheta}=-\frac{g}{\ell} \sin \vartheta \\
\vartheta(0)=\vartheta_{0} \neq 0 \\
\dot{\vartheta}_{0}=0
\end{array}\right.
$$

Putting as usual:

$$
\left\{\begin{array}{l}
q=\vartheta \\
p=\dot{\vartheta}
\end{array}\right.
$$

we obtain the pendulum Hamiltonian:

$$
H(p, q)=\frac{1}{2} p^{2}+\frac{g}{\ell}(1-\cos q),
$$


which supplies the sistem energy amount as a function of the nonzero initial condition $\vartheta_{0}$ :

$$
H(p, q)=2 \frac{g}{\ell} \sin ^{2} \frac{\vartheta_{0}}{2}:=h .
$$

The motion period depends on the complete first kind elliptic integral:

$$
T=4 \sqrt{\frac{\ell}{g}} \mathbf{K}\left(\sin \frac{\vartheta_{0}}{2}\right) .
$$

As a consequence, comparing the expansion of the elliptic integral (13) with that given by our Theorem (1.2), we are going to obtain a remarkable validation of our approach. Let us remember the complete elliptic integral's expansion:

$$
\begin{aligned}
\mathbf{K}\left(\sin \frac{\vartheta_{0}}{2}\right) \sim & \frac{\pi}{2}\left(1+\frac{1}{4} \sin ^{2} \frac{\vartheta_{0}}{2}+\frac{9}{64} \sin ^{4} \frac{\vartheta_{0}}{2}+\frac{25}{256} \sin ^{6} \frac{\vartheta_{0}}{2}+\right. \\
& +\frac{1,225}{16,384} \sin ^{8} \frac{\vartheta_{0}}{2}+\frac{3,969}{65,536} \sin ^{10} \frac{\vartheta_{0}}{2}+ \\
& \left.+\frac{53,361}{1,048,576} \sin ^{12} \frac{\vartheta_{0}}{2}+\frac{184,041}{4,194,304} \sin ^{14} \frac{\vartheta_{0}}{2}+\cdots\right) .
\end{aligned}
$$

On the other hand, applying the reversion to $x=G(q)=\frac{g}{\ell}(1-\cos q)$, we obtain:

$$
\begin{aligned}
q= & \sqrt{\frac{2 \ell}{g}}\left(x^{\frac{1}{2}}+\frac{\ell}{12 g} x^{\frac{3}{2}}+\frac{3 \ell^{2}}{160 g^{2}} x^{\frac{5}{2}}+\frac{5 \ell^{3}}{896 g^{3}} x^{\frac{7}{2}}+\frac{35 \ell^{4}}{18,432 g^{4}} x^{\frac{9}{2}}\right. \\
& \left.+\frac{63 \ell^{5}}{90,112 g^{5}} x^{\frac{11}{2}}+\frac{231 \ell^{6}}{851,968 g^{6}} x^{\frac{13}{2}}+\frac{143 \ell^{7}}{1,310,720 g^{7}} x^{\frac{15}{2}}+\cdots\right)
\end{aligned}
$$

whilst the reversion of $x=F(p)=\frac{1}{2} p^{2}$ gives $p=(2 x)^{\frac{1}{2}}$. Therefore, applying Theorem (1.2) we conclude that period can asymptotically be expanded as a function of the energy level $h=2 \frac{g}{\ell} \sin ^{2} \frac{\theta_{0}}{2}$ :

$$
\begin{aligned}
T(h)= & \frac{\pi}{2} \sqrt{\frac{\ell}{g}}\left(4+\sin ^{2} \frac{\vartheta_{0}}{2}+\frac{9}{16} \sin ^{4} \frac{\vartheta_{0}}{2}+\frac{25}{64} \sin ^{6} \frac{\vartheta_{0}}{2}+\frac{1,225}{4,096} \sin ^{8} \frac{\vartheta_{0}}{2}+\right. \\
& \left.+\frac{3,969}{16,384} \sin ^{10} \frac{\vartheta_{0}}{2}+\frac{53,361}{262,144} \sin ^{12} \frac{\vartheta_{0}}{2}+\frac{184,041}{1,048,576} \sin ^{14} \frac{\vartheta_{0}}{2}+\cdots\right)
\end{aligned}
$$

Multiplying (14) to $4 \sqrt{\frac{\ell}{g}}$, the asymptotic expansion supplied by Theorem (1.2) is exactly found. Therefore Theorem (1.2) gives another path for arriving at Legendre's expression of the complete first kind elliptic integral.

\subsection{Duffing equation}

Let $\varepsilon>0$, then the Duffing equation:

$$
\left\{\begin{array}{l}
\ddot{x}+x+\varepsilon x^{3}=0, \\
x(0)=a \neq 0, \\
\dot{x}(0)=0 .
\end{array}\right.
$$

models free oscillations induced by a non linear elastic element. This equation belongs to systems we are tackling being the Hamiltonian:

$$
H(p, q)=\frac{1}{2} p^{2}+\frac{1}{2} q^{2}+\frac{1}{4} \varepsilon q^{4}
$$

uncoupled. The energy level is: 


$$
H(p, q)=\frac{1}{2} a^{2}+\frac{1}{4} \varepsilon a^{4}:=h .
$$

Equation (15) can be integrated via elliptic functions, see [13], and solution's period computed by:

$$
T=\frac{4}{\sqrt{1+\varepsilon a^{2}}} \mathbf{K}\left(\frac{\varepsilon a^{2}}{2+2 \varepsilon a^{2}}\right) .
$$

Let us remember again the $\mathbf{K}$ elliptic integral expansion:

$$
\mathbf{K}(a) \sim \pi\left(\frac{1}{2}+\frac{1}{8} a+\frac{9}{128} a^{2}+\frac{25}{512} a^{3}+\frac{1,225}{32,768} a^{4}+\frac{3,969}{131,072} a^{5}+\cdots\right),
$$

replacing $a$ with $\frac{\varepsilon a^{2}}{2\left(1+\varepsilon a^{2}\right)} \sim \frac{a^{2} \varepsilon}{2}-\frac{a^{4} \varepsilon^{2}}{2}+\frac{a^{6} \varepsilon^{3}}{2}-\frac{a^{8} \varepsilon^{4}}{2}+\frac{a^{10} \varepsilon^{5}}{2}+\cdots$ we get:

$$
\begin{aligned}
\mathbf{K}\left(\frac{\varepsilon a^{2}}{2\left(1+\varepsilon a^{2}\right)}\right) \sim & \frac{\pi}{2}+\frac{a^{2} \pi \varepsilon}{16}-\frac{23 a^{4} \pi \varepsilon^{2}}{512}+\frac{137 a^{6} \pi \varepsilon^{3}}{4,096}+ \\
& -\frac{13,495 a^{8} \pi \varepsilon^{4}}{524,288}+\frac{85,601 a^{10} \pi \varepsilon^{5}}{4,194,304}+\cdots
\end{aligned}
$$

and taking into account that:

$$
\frac{4}{\sqrt{1+\varepsilon a^{2}}} \sim 4-2 a^{2} \varepsilon+\frac{3}{2} \varepsilon^{2} a^{4}-\frac{5}{4} \varepsilon^{3} a^{6}+\frac{35}{32} \varepsilon^{4} a^{8}-\frac{63}{64} \varepsilon^{5} a^{10}+\cdots
$$

we finally obtain the periodal asymptotic expansion:

$$
\begin{aligned}
T \sim & \pi\left(2-\frac{3}{4} \varepsilon a^{2}+\frac{57}{128} \varepsilon^{2} a^{4}-\frac{315}{1,024} \varepsilon^{3} a^{6}+\right. \\
& \left.+\frac{30,345}{131,072} \varepsilon^{4} a^{8}-\frac{193,347}{1,048,576} \varepsilon^{5} a^{10}+\cdots\right)
\end{aligned}
$$

The reversion of function $\widetilde{G}(q)=G(q)+G(-q)=x$ is:

$$
\sqrt{2}\left(2 x^{\frac{1}{2}}-2 \varepsilon x^{\frac{3}{2}}+\frac{7}{4} \omega^{2} x^{\frac{5}{2}}-\frac{33}{8} \varepsilon^{3} x^{\frac{7}{2}}+\frac{715}{64} \varepsilon^{4} x^{\frac{9}{2}}+\cdots\right) .
$$

Then Theorem (1.2) gives period asymptotic expansion:

$$
\begin{aligned}
T(h) \sim & \pi\left(2-\frac{3}{2} \varepsilon h+\frac{105}{32} \varepsilon^{2} h^{2}-\frac{1,155}{128} \varepsilon^{3} h^{3}+\right. \\
& \left.+\frac{225,225}{8,192} \varepsilon^{4} h^{4}-\frac{2,909,907}{32,768} \varepsilon^{5} h^{5}+\cdots\right) .
\end{aligned}
$$

Replacing the $h$ value given by (16) in (18), neglecting the $a$ powers of exponent $>10$, we will find (17) again. Therefore we checked once more that the period approximation supplied by Theorem (1.2), agrees with otherwise computed results.

\subsection{Elastic wire-mass system}

Let us take the Hamiltonian:

$$
H(p, q)=\frac{1}{2 m} p^{2}+\left(\frac{1-2 b}{2}-\frac{-2 b-2 b q^{2}+\sqrt{1+q^{2}}+q^{2} \sqrt{1+q^{2}}}{2 \sqrt{1+q^{2}}}\right) \omega^{2}
$$

introduced in [1] with $m>0, b>1$ and $\omega^{2}=\frac{k}{m}$. Such a system consists of a particle of mass $m$ flowing frictionless along a direction $O x$, under the continuous effect of an elastic wire of constant $k$ linking it to a 
fixed point belonging to the vertical axis $O y$ at height 1 . Assumption $b>1$ ensures system periodic behaviour, implying $G(q)$ have a minimum at the origin. Nevertheless its oscillation period - as far as we are concerned - cannot be expressed even through special functions. Theorem (1.2) being highly general, makes up for the lack, supplying period function asymptotically via reversion:

$$
\begin{aligned}
T(h) \sim & \pi\left(\frac{2}{(b-1)^{\frac{1}{2}} \omega}+\frac{3 b}{4(b-1)^{\frac{5}{2}} \omega^{3}} h-\frac{15(b-8) b}{128(b-1)^{\frac{9}{2}} \omega^{5}} h^{2}+\right. \\
& \left.+\frac{35 b\left(b^{2}-8 b+40\right)}{1,024(b-1)^{\frac{13}{2}} \omega^{7}} h^{3}+\cdots\right) .
\end{aligned}
$$

\section{Conclusions}

By Theorem (1.2) the energy-period functions of a broad class of Hamiltonian systems are allowed to be expanded asymptotically for a wide class of single - degree of freedom non dissipative Hamiltonian systems.

It has been validated with some Hamiltonian systems otherwise known.

It is true that uniform convergency of the power series obtained via our asymptotic expansion can be deemed true being the energy - period function coming from an integration. Nevertheless, a direct proof of it is highly difficult being the power series coefficients determined by two reversions and then usual methods for checking the uniform convergency are out of practice.

The example of the wire-mass system fully explains the power of our approach which enables to obtainunder our assumptions-the oscillation period expansion even for those systems whose time equation cannot be integrated in closed form.

All the relevant symbolic computations described throughout this paper have been performed by means of Mathematica $\mathbb{R}$ version 4.1 of Wolfram Research implemented on PowerPC Apple, processor G4, 733 MHz.

\section{acknowledgements}

Research supported by MURST grant: Metodi matematici in economia

\section{References}

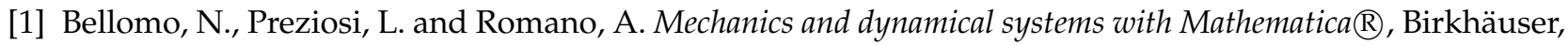
Boston 2000.

[2] Chicone, C. and Jacobs, M. 'Bifurcation of critical periods for plane vector fields', Trans. Am. Math. Soc. 312 (1989), 433-486.

[3] Chicone, C. 'The Monotonicity of the Period Funcion for Planar Hamiltonian Vector Fields', J. Differential Equations 69, (1987), 310-321.

[4] Cima, A., Gasul, A. and Mañosas, F. 'Period Function for a Class of Hamiltonian Systems', J. Differential Equations 168, (2000) 180-199.

[5] Cima, A., Mañosas, F. and Villadeprat, J. 'Isochronicity for Several Classes of Hamiltonian Systems', J. Differential Equations 157, (1999), 373-413.

[6] Compton, E.T. Asymptotic Expansions, Cambridge University Press, Cambridge, 1965.

[7] Coppel, W. A. and Gavrilov, L. 'The period of a Hamiltonian quadratic system', Diff. Int. Eq. 6, (1993), 1357-1365.

[8] Erdèlyi, A. Asymptotic Expansions, Dover, New York, 1956.

[9] Gasull, A., Guillamon, A., Mañosa,V. and Mañosas, F. 'The Period Function for Hamiltonian Systems with Homogeneous Nonlinearities', J. Differential Equations 139, (1997), 237-260. 
[10] Gasull, A., Guillamon, A. and Mañosa, V. 'An Explicit Expression of the first Liapunov and Period Constants with Applications', J. Math. Anal. Appl. 211, (1997), 190-212.

[11] Herz, A. V. M. 'Solutions of $\dot{x}(t)=-g(x(t-1))$ Approach the Kaplan-Yorke Orbits for Odd Sigmoid $g^{\prime}$, J. Differential Equations 118, (1995), 36-53.

[12] Knopp, K., Theory and application of infinite series, Blackie - Son, London, 1928, reprinted by Dover, New York, 1990.

[13] Lawden, D.F., Elliptic Functions and Applications, Springer, New York, 1989.

[14] Nayfeh, A. H., Perturbation methods, John Wiley and sons, New York, 1973.

[15] Olver, F.W.J. Asymptotics and Special Functions, Academic Press, New York 1974.

[16] Rothe, F. 'The periods of the Volterra-Lotka system', J. Reine Angew. Math., 355, (1985), 129-138.

[17] Rothe, F. 'Remarks on periods of planar Hamiltonian systems' Siam J. Math. Anal., 24, (1993), n. 1, $129-154$.

[18] Schaaf, R. 'Global behaviour of solution branches for some Neumann problems depending on one or several parameters', J. Pure Appl. Math. 147, (1984), 1-31.

[19] Uribe, M. and Wallace, S.M., 'The Period Function in a class of Quadratic Kolmogoroff Systems', Proyecciones 19, (2000), 197-205.

[20] Whittaker, E.T. and Watson, G.N. A Course of Modern Analysis, Cambridge University Press, Cambridge, 1927. 